\title{
Stress effects on stability and diffusion behavior of sulfur impurity in nickel: A first-principles study
}

\author{
Nan Dong ${ }^{\mathrm{a}, \mathrm{b}}$, Caili Zhang ${ }^{\mathrm{a}, \mathrm{b}}$, Hui Liu ${ }^{\mathrm{a}, \mathrm{b}}$, Juan $\mathrm{Li}^{\mathrm{a}, \mathrm{b}}$, Xiaolei $\mathrm{Wu}^{\mathrm{c}}$, Peide Han ${ }^{\mathrm{a}, \mathrm{b}, *}$ \\ ${ }^{a}$ Key Laboratory of Interface Science and Engineering in Advanced Materials, Taiyuan University of Technology, Ministry of Education, Taiyuan 030024, China \\ ${ }^{b}$ College of Materials Science and Engineering, Taiyuan University of Technology, No. 79 Yingze Street, Wanbolin District, Taiyuan 030024, China \\ ' State Key Laboratory of Nonlinear Mechanics, Instite of Mechanics, Chinese Academy of Sciences, Beijing 100080, China
}

\section{A R T I C L E I N F O}

\section{Article history:}

Received 8 July 2013

Received in revised form 4 April 2014

Accepted 5 April 2014

Available online 4 May 2014

\section{Keywords:}

Nickel

Sulfur

First-principles

Diffusion

Stress effect

\begin{abstract}
A B S T R A C T
A systematic investigation regarding the effect of stress on the stability and diffusion behavior of S impurity in $\mathrm{Ni}$ was carried out via first-principles methods. A comparison of the formation energy of $\mathrm{S}$ in $\mathrm{Ni}$ indicated that $\mathrm{S}$ more easily forms as a solution atom with increasing $\mathrm{S}$ concentration in Ni supercells, but the binding energy showed that as the concentration of $\mathrm{S}$ that dissolved into Ni increased, the structure became less stable. The diffusion barrier via the octahedral-tetrahedral-octahedral site path was always lower than that via the octahedral-octahedral site path. The diffusion barrier of single S decreased with increase in tensile stress. S diffusion accelerated under applied tensile stress, which was disadvantageous in suppressing $\mathrm{S}$ retention in $\mathrm{Ni}$. These results implied that even at a low concentration, dissolved $\mathrm{S}$ still had a tendency of precipitating from the Ni matrix, to further increase the stability of the system. (c) 2014 Elsevier B.V. All rights reserved.
\end{abstract}

\section{Introduction}

Nickel $(\mathrm{Ni})$ is a crucial element for use in high-temperature structural materials because of its applications in high-performance bellows and combustion chambers with cooling tubes used in spacecraft and aircraft [1-3]. Understanding the effects of alloying elements and impurities on the mechanical properties and corrosion resistance of $\mathrm{Ni}$-based alloys is necessary to maintain the functionality of these alloys in high-temperature environments [4-7]. However, the reliability of Ni-based alloys as structural materials is limited by the brittleness induced by impurity segregation during service at high temperatures $[8,9]$. Some solute elements segregating on grain boundaries (GBs) can weaken the bonding of GBs. Despite the long and continuing effort to understand sulfur segregation to GB embrittlement of $\mathrm{Ni}$ [10-14], the physics and chemistry underlying the phenomenon remain unclear. Electrodeposited nanocrystalline Ni contains impurities such as carbon, oxygen and sulfur $[15,16]$. Sulfur has a high-grain boundary enrichment ratio to $\mathrm{Ni}\left(\sim 10^{4}\right)$ because of its extremely low solid solubility $\left(<10^{-4}\right)$ to Ni. Lozinskiy reported that hightemperature embrittlement occurs at temperatures ranging from $773 \mathrm{~K}$ to $1273 \mathrm{~K}$ when sulfur content in $\mathrm{Ni}$ is $>20 \mathrm{ppm}$. This embrit-

\footnotetext{
* Corresponding author at: College of Materials Science and Engineering, Taiyuan University of Technology, No. 79 Yingze Street, Wanbolin District, Taiyuan 030024 China. Tel.: +86351 6018843; fax: +863516014208.

E-mail addresses: hanpeide@126.com, hanpeide@tyut.edu.cn (P. Han).
}

tlement becomes significant with increasing sulfur content. Moreover, the embrittlement takes place even at approximately room temperature for sulfur content $>140 \mathrm{ppm}$ [17]. Considering the segregation of sulfur to the GBs of $\mathrm{Ni}$, the latter becomes more fragile and particularly susceptible to in situ fracturing. Despite decades of intense experimental and theoretical efforts, a direct and fundamental study focusing on $\mathrm{S}$ trapping and blistering in $\mathrm{Ni}$ at the atomic level is still lacking [13,18-21]. The interaction of S with metals and metal alloys has elicited considerable scientific and technological interest. Although the presence of these impurities is interesting, it also has a profound effect on the tensile and creep behavior of nanocrystalline Ni. Matsui et al. considered the possibility that the increase in the hardness of nanocrystalline $\mathrm{Ni}$ is due to the solid solution strengthening induced by $\mathrm{C}$ and $\mathrm{S}$ $[8,16,22]$.

The present contribution focuses on the effect of grain boundary embrittlement of sulfur segregation at a nickel grain boundary $[23,24]$. Precipitation and segregation to GBs are related to the diffusion barrier of the elements. However, the reason and the mechanism behind the precipitation and segregation into the GBs of a critical intergranular sulfur concentration remain unclear. To date, no research on the stability and diffusion behavior of $\mathrm{S}$ in $\mathrm{Ni}$ under stress has been reported. Therefore, examination of the stability of $\mathrm{S}$ in $\mathrm{Ni}$ is crucial. To understand the physical mechanism underlying the interaction of $\mathrm{S}$ with $\mathrm{Ni}$, this study presents for the first time the structure, stability, and diffusion behavior of $\mathrm{S}$ in $\mathrm{Ni}$ using first-principles calculations. 


\section{Calculation method and model}

\subsection{Calculation method}

First-principles calculations were performed using the CASTEP code (Cambridge Sequential Total Energy Package) based on density functional theory (DFT) $[15,25]$. We used the PerdewBurke-Ernzerh (PBE) of function within the generalized gradient approximation. The states of $\mathrm{Ni} 3 \mathrm{~d}_{8} 4 \mathrm{~s}_{2}$ and $\mathrm{S} 3 \mathrm{~s}_{2} 3 \mathrm{p}_{4}$ were treated as valence states. Ultrasoft pseudopotentials, known for their high efficiency in calculating structural and electronic properties, were expanded within a plane wave basis set works. A kinetic energy cutoff of $300 \mathrm{eV}$ was used after carefully testing for convergence when the geometry of the system was changed during slip calculations and when $S$ impurities were included. Finally, a uniform k-point grid of $6 \times 6 \times 6$ for the Brillouin zone was used for the fcc bulk Ni calculations with four atoms in the cubic unit cell. The convergence parameters were as follows: total energy tolerance, $1.0 \times 10^{-5} \mathrm{eV} /$ atom; maximum force tolerance, $0.3 \mathrm{eV} / \mathrm{nm}$; maximal stress component, $0.03 \mathrm{GPa}$; and maximal displacement, $1 \times 10^{-4} \mathrm{~nm}$.

To determine the effect of stress on stability and diffusion behavior of $\mathrm{S}$ in $\mathrm{Ni}$, a uniaxial strain was introduced and the supercell was stretched by a small increment parallel to the $z$-axis. The atomic basis vectors perpendicular to the applied strain were simultaneously relaxed. The application of $z$-axis strain (either tensile or compressive) on $\mathrm{Ni}$ could directly reflect the stress effect. The range of applied strain was between $-5 \%$ and $+5 \%$ with an interval of $1 \%$. The drag method was employed to determine the diffusion barrier of single $S$ under applied stress. We fixed the volume and constrained the atomic positions to relax in a hyper plane perpendicular to the vector from the initial to the final position [26] to obtain the diffusion barrier for the strain, and the numbers of equal separated intervals for the $\mathrm{O}-\mathrm{O}$ path and the $\mathrm{O}-\mathrm{T}-\mathrm{O}$ path are 5 and 10 . We defined the diffusion barrier as the energy maximum values minus the energy minimum values in each diffusion path.

The formation energy [27] of S atoms in intrinsic Ni can be defined by Eq. (1):

$E^{f}=\left(m E_{\text {solid }}^{N i}+n E_{\text {solid }}^{S}-E_{\text {tot }}^{\left(N i_{m} S_{n}\right)}\right) / X$

where the $E_{\text {solid }}^{N i}$ is the energy of pure element Ni per atom, $E_{\text {solid }}^{S}$ is the energy of pure element $S$ per atom. $E_{t o t}^{\left(N i_{m} S_{n}\right)}$ is the total energy of the supercell containing $m \mathrm{Ni}$ atoms and $n \mathrm{~S}$ atoms. The energy of pure elements was calculated by using their ground state conventional cell as fcc for $\mathrm{Ni}$ and $\alpha \mathrm{S}-\mathrm{oF} 128$ for $\mathrm{S}$. $X$ is the number of the cell in the calculation model.

The term "binding energy" the energy is released upon the creation of a bound state and which is used to compare with the relative stability of structure and thermodynamic properties. The binding energy of S atoms in intrinsic Ni can be defined by Eq. (2):

$E^{b}=\left(m E_{\text {atom }}^{N i}+n E_{\text {atom }}^{S}-E_{\text {tot }}^{\left(\mathrm{Ni}_{m} S_{n}\right)}\right) /(n+m)$

where the $E_{\text {atom }}^{N i}$ is the energy of an isolated Ni atom, $E_{\text {atom }}^{S}$ is the energy of an isolated $\mathrm{S}$ atom. $E_{\text {tot }}^{\left(\mathrm{Ni}_{m} S_{n}\right)}$ is the total energy of the supercell containing $m \mathrm{Ni}$ atoms and $n \mathrm{~S}$ atoms. The total energies of isolated atoms were calculated by putting a $\mathrm{Ni}$ or $\mathrm{S}$ atom in the middle of a lattice constant of $10 \AA$ cubic unit cell, and then taken from the CASTEP output files directly.

\subsection{Calculation model}

Nickel is a face-centered cubic (fcc) that occupies the position of vertex and vortex. The space group of $\mathrm{Ni}$ is $\mathrm{P} 42 / \mathrm{mnm}$, and the crystal symmetry is $\mathrm{D}_{4 \mathrm{~h}-14}$. Using the current theoretical scheme, the calculated lattice constant of fcc Ni solid is $3.5586 \AA$, which is in good agreement with the experimental value of $3.5240 \AA$ [28]. Within fcc meftal, two types of typical point defects exist: substitutional and interstitial. $\mathrm{Ni}_{8}$ and $\mathrm{Ni}_{7} \mathrm{~S}$ substitutional solid solutions were modeled using $1 \times 1 \times 2$ super cells $(X=2)$, which were periodic in all three spatial directions and contain eight atoms (Fig. 1a and b). As illustrated in Fig. 1c and d, the possible interstitial sites include octahedral interstitial sites (OIS) and tetrahedral interstitial sites (TIS), respectively. The $\mathrm{Ni}_{8} \mathrm{~S}$ supercells contain one interstitial solute atom per supercell. Within our supercell model, the formation energy and the binding energy could be obtained by four fcc supercells: $1 \times 1 \times 1\left(\mathrm{Ni}_{4} \mathrm{~S}, X=1\right), 1 \times 1 \times 2\left(\mathrm{Ni}_{8} \mathrm{~S}, X=2\right), 1 \times 2 \times 2$ $\left(\mathrm{Ni}_{16} \mathrm{~S}, X=4\right)$ and $2 \times 2 \times 2\left(\mathrm{Ni}_{32} \mathrm{~S}, X=8\right)$. These supercells correspond to $20,11.11,5.88$ and 3.03 at.\% interstitial solid solutions.

\section{Results and discussion}

\subsection{Stable position of $\mathrm{S}$ in $\mathrm{Ni}$}

We first examined the stability of a single $S$ impurity in possible interstitial and substitutional positions of the fcc Ni solid. For comparison, we hypothesized that the $S$ atom was at the center of substitution site and the interstitial sites. In order to guarantee the $S$ atom was at the OIS and TIS, we constrain the $S$ and neighboring $\mathrm{Ni}$ atomic positions during relaxing. The cell parameters, formation energy and binding energy for the substitutional, OIS, and TIS were calculated and listed in Table 1. Table 1 shows that the insertions of $\mathrm{S}$ into $\mathrm{Ni}$ caused the increase of the distance between atoms and the volume, and in substitutional site produced the smallest volume change. For a single impurity of $\mathrm{S}$, the most easily formed position was the substitutional site with corresponding formation energy of $1.1040 \mathrm{eV}$. All formation energies were positive, indicating that the incorporation of $\mathrm{S}$ impurities into Ni host was always an exothermic process. The binding energy of the bulk $\mathrm{Ni}\left(\mathrm{Ni}_{8}\right)$, substitutional site $\left(\mathrm{Ni}_{7} \mathrm{~S}\right)$, OIS $\left(\mathrm{Ni}_{8} \mathrm{~S}\right)$, and TIS $\left(\mathrm{Ni}_{8} \mathrm{~S}\right)$ were $4.1329 \mathrm{eV}, 4.1120 \mathrm{eV}, 3.9168 \mathrm{eV}$ and $3.8568 \mathrm{eV}$, respectively. These showed that the binding energy of the bulk Ni was larger than that of $\mathrm{S}$ in Ni indicating that the system became less stable after $\mathrm{S}$ insertion. In addition, the distance between different atoms (in Fig. 1): $d_{1-3}$ (Nos. 1 and 3), $d_{2-3}$ (Nos. 2 and 3 ) and $d_{1-4}$ (Nos. 1 and 4) of the substitutional site, OIS, and TIS became larger than that of the bulk $\mathrm{Ni}$ showed that the bonding between $\mathrm{Ni}$ atoms became weaker, finally decreased the stability of the structure.

Using the simulation lattice parameter of fcc Ni (3.5586 $\AA$ ), the radii of the OIS and TIS were 0.5215 and $0.2828 \AA$, respectively. $S$ had a covalent radius of $1.04 \AA$, suggesting that it can fit better in the OIS. Considering that the substitutional sites include the process of kicking one Ni atom out of the perfect crystal. This case is

Table 1

Calculated lattice parameters, formation energy and binding energy for single $\mathrm{S}$ atom in different sites in $\mathrm{fcc} \mathrm{Ni}_{8}$.

\begin{tabular}{lllll}
\hline Position & Clean & Sub & OIS & TIS \\
\hline$a / \AA$ & 3.5586 & 3.5385 & 3.9190 & 4.0459 \\
$b / \AA$ & 3.5586 & 3.5321 & 3.9190 & 4.0459 \\
$c / \AA$ & 7.1242 & 7.4674 & 7.6899 & 8.0357 \\
$\alpha /^{\circ}$ & 90.0001 & 90 & 90 & 90 \\
$\beta /{ }^{\circ}$ & 89.9999 & 90 & 90 & 90 \\
$\gamma /{ }^{\circ}$ & 90 & 90 & 90 & 90 \\
$V / \AA^{3}$ & 90.2191 & 93.3298 & 118.1071 & 131.5388 \\
$d_{1-3} / \AA$ & 2.516 & 2.654 & 2.745 & 2.888 \\
$d_{2-3} / \AA$ & 2.516 & 2.584 & 2.771 & 2.901 \\
$d_{1-4} / \AA$ & 2.516 & 2.584 & 2.771 & 2.901 \\
$E_{f}(\mathrm{eV})$ & 0 & 1.1040 & 0.4248 & 0.1275 \\
$E_{b}(\mathrm{eV})$ & 4.1329 & 4.1120 & 3.9168 & 3.8568 \\
\hline
\end{tabular}




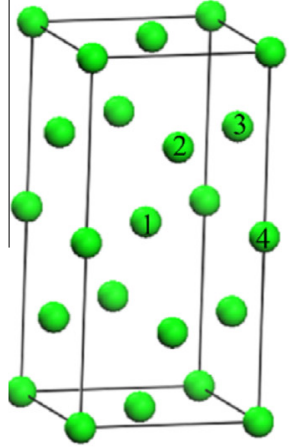

(a)

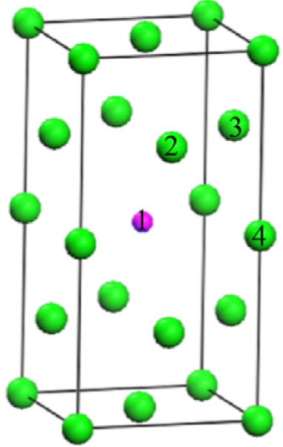

(b)

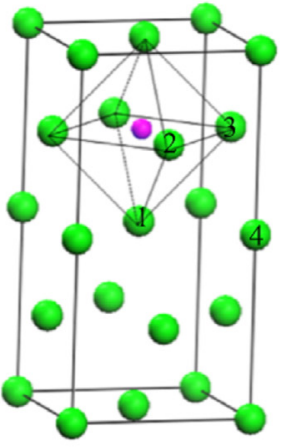

(c)

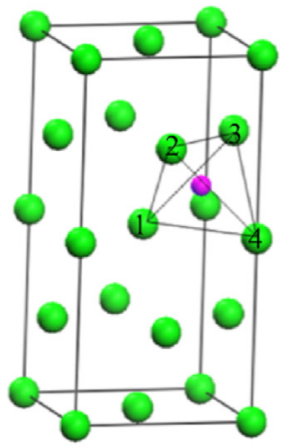

(d)

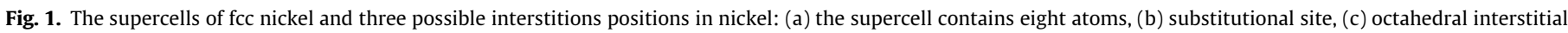

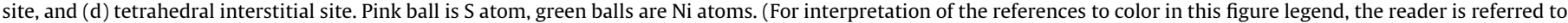
the web version of this article.)
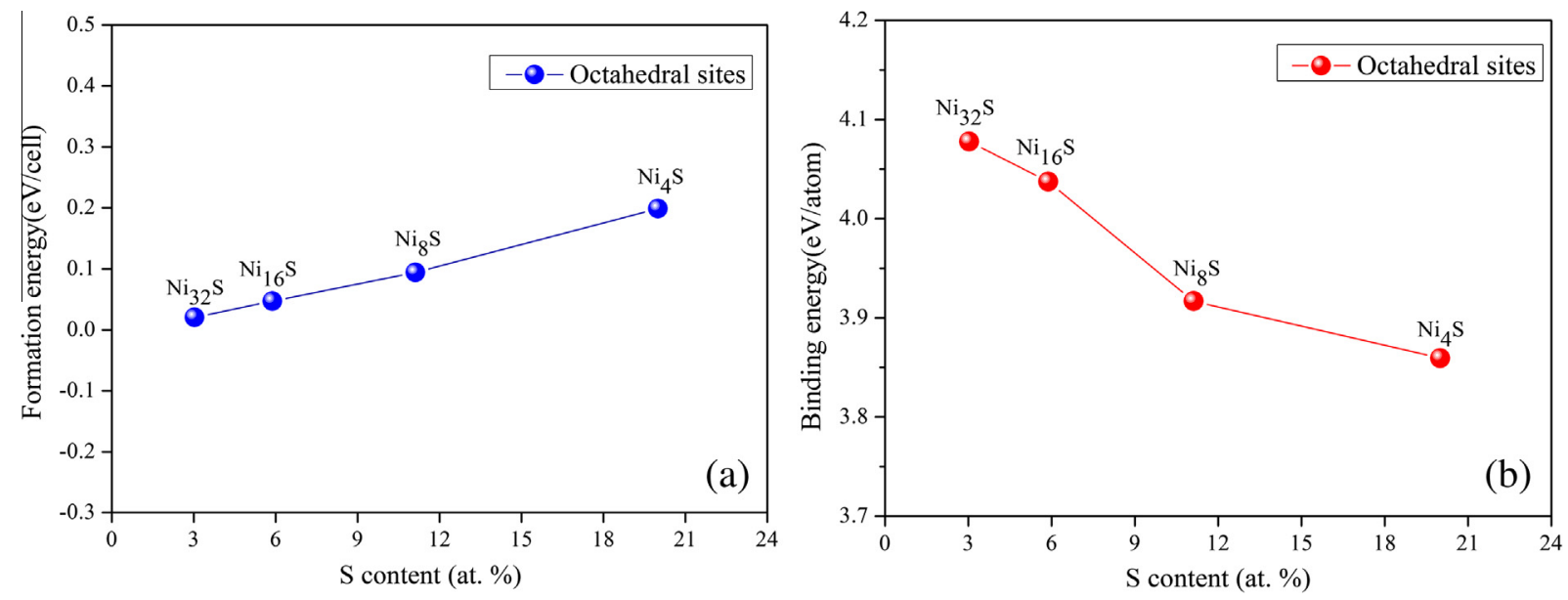

Fig. 2. The formation energy and the binding energy of different concentration of S dissolve into Ni. (a) The formation energy. (b) The binding energy.

much harder than those of interstitial cases. So we calculated the formation and binding energies of different $\mathrm{S}$ concentrations at OIS in supercells of Ni (Fig. 2). As shown in Fig. 2a, the formation energy increased with increasing $\mathrm{S}$ concentration in Ni supercells, suggesting that the formation of S-doped $\mathrm{Ni}$ became easier. The stability of S-doped $\mathrm{Ni}$ is related to its binding energies. On the contrary, the binding energy decreased as the concentration of $\mathrm{S}$ that dissolved into $\mathrm{Ni}$ increased, showing that the stability of the $\mathrm{S}$-doped Ni became more difficult as shown in Fig. $2 \mathrm{~b}$. This result indicated that even at such a low concentration (3.03 at.\%), dissolved $\mathrm{S}$ still had a tendency to precipitate from the Ni host to increase the stability of the system further [29]. S would quickly migrate trapping sites, which could be vacancies, grain boundaries, or dislocations. In this case, even small concentrations of $\mathrm{S}$ could act as strong embrittlers because the local concentration at crystalline defects could be high [10-14].

The charge density distribution reflects the bonding characteristics between atoms [30]. We determined any electronic factors contributing to the relation between $\mathrm{S}$ and $\mathrm{Ni}$. Fig. $3 \mathrm{a}$ and $\mathrm{b}$ display the charge density distribution maps in the (002) plane of $\mathrm{Ni}_{4}$ and $\mathrm{Ni}_{4} \mathrm{~S}$ with the $\mathrm{S}$ in OIS, respectively. For both cases, the area with lower electron density (the yellow ${ }^{1}$ area) became larger with $S$ into $\mathrm{Ni}$. The electron localization around the nucleus of $\mathrm{Ni}$ and the

\footnotetext{
${ }^{1}$ For interpretation of color in Figs. 3 and 5, the reader is referred to the web version of this article.
}

neighboring $\mathrm{Ni}$ atom remained near zero. As clearly shown in Fig. 3b, a significant charge transfer occurred between $\mathrm{S}$ and $\mathrm{Ni}$. The charge density was higher between $\mathrm{S}$ and Ni than that with pure Ni. This phenomenon suggests that the $\mathrm{S}$ atom exhibited interaction with the surrounding lattice atoms.

We further examined the charge transfer between $\mathrm{S}$ and $\mathrm{Ni}$ using Bader charge analysis based on atoms in molecular theory with a grid-based algorithm [31,32]. In this analysis, the real space was partitioned into several subspaces associated with each atom based on the charge density distribution. The boundary of an atom was delimited by the zero-flux surface of the charge density gradient vector field upon which the charge density reached minimum perpendicular to the surface. The integrated charge enclosed within the zero-flux surfaces can be taken as a good approximation of the charge of an atom. Fig. $3 \mathrm{c}$ and d presented the Bader charge of $\mathrm{Ni}_{4}$ and $\mathrm{Ni}_{4} \mathrm{~S}$, respectively. For $\mathrm{Ni}_{4}$, all Bader charges of $\mathrm{Ni}$ atoms at the position of vertex and vortex (Nos. 1, 2, 3, 4, 5, and 6) were 0 e. For the OIS case, the Bader charge of $\mathrm{Ni}$ at the position of vertex was $0.26 \mathrm{e}$. The interstitial $\mathrm{S}$ atom obtained the electron from the host $\mathrm{Ni}$ atom at the position of vertex and became negatively charged, with a Bader charge of approximately -0.05 e. The Bader charges of $\mathrm{Ni}$ at the position of vortex (Nos. 1 and 3), Ni (Nos. 2 and 4 ), and Ni (Nos. 5 and 6) were $-0.09,-0.09$, and -0.03 e, respectively. The calculated Bader charge also reveals that $\mathrm{S}$ induces a slight reduction in the directionality of bonding between $\mathrm{Ni}$ atoms. The system is meeting the charge transfer conservation. From Fig. $3 c$ and $d$, we also see that the insertion of $\mathrm{S}$ into Ni caused 

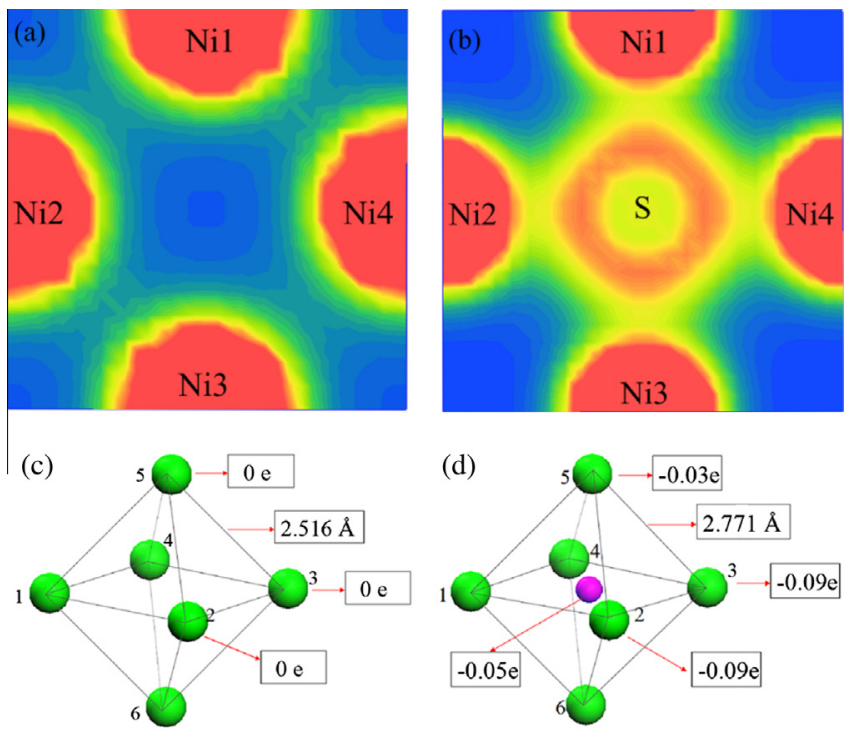

(e)

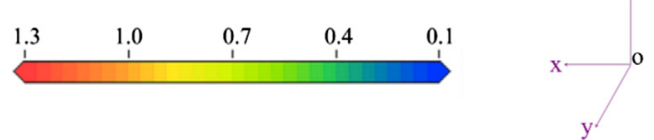

Fig. 3. The charge density maps along the (002) plane, for the cases of (a) $\mathrm{Ni}_{4}$, (b) $\mathrm{S}$ in OIS. Isosurface values: from 0.1 to 1.3 . The Bader charges for (c) $\mathrm{Ni}_{4}$, (d) $\mathrm{S}$ in OIS.

the increase of the distance between atoms $d_{1-2}, d_{2-3}, d_{3-4}, d_{4-1}, d_{5-}$ $1, d_{5-2}, d_{5-3}, d_{5-4}, d_{6-1}, d_{6-2}, d_{6-3}$ and $d_{6-4}$ from $2.516 \AA$ to $2.771 \AA$, weaken the bonding of the $\mathrm{Ni}$ atoms around the $\mathrm{S}$ atom, which is in good agreement with the result that we have seen before.

\subsection{Stability and charge density distribution under stress}

As shown in Section 3.1, the S atom was not energetically favorable sitting at any other site. Thus, we calculated the total energy of single $S$ at two interstitial cases (TIS and OIS) to investigate the site preference of single $S$ atom under $z$-axis stress. The total energy at the OIS and TIS without strain was -5685.0508 and -5673.9557 $\mathrm{eV}$, respectively, showing that S preferred the OIS site. Consistent with the experimental observations, the negative binding energy indicated that dissolution of $\mathrm{S}$ in $\mathrm{Ni}$ is an exothermic process [16].

We showed the relative values of the total energy of single $S$ atom at the TIS and OIS using the total energy of OIS as the reference as a function of strain in Fig. 4. The relative energy value decreased for both the TIS and OIS with increasing tensile stress or decreasing compressive stress. This result indicates that the compressive stress made the $\mathrm{S}$ solution in Ni more difficult, whereas the tensile stress made the solution easier. In other words, the tensile stress promoted structure stability, whereas the compressive stress promoted structure instability. The vacant space for $\mathrm{S}$ occupation will be smaller because of the compression, leading to higher energy [33]. Furthermore, the relative values of the total energy at the TIS were larger than those of the total energy at the OIS. Namely, the applied stress did not change the relative stability of $\mathrm{S}$ in $\mathrm{Ni}$.

Fig. 5a-f shows the valence charge density distributions of the (020) plane (Fig. 5a-c) and the (002) plane (Fig. 5d-f) of single $S$ atom at the OIS with a compressive strain of $-5 \%$, without strain, and a tensile strain of $+5 \%$, respectively. Correspondingly, Fig. 5 shows that the presence of the $z$-axis stress changes the electron distribution. Moreover, the area with lower electron density (the yellow area) becomes larger between $\mathrm{Ni}$ and $\mathrm{S}$ with increasing strain from compressive to tensile.

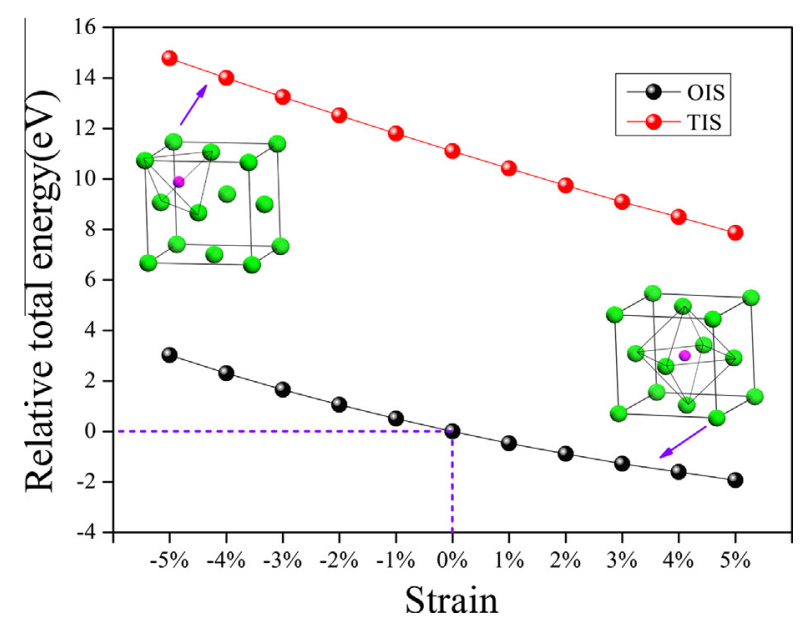

Fig. 4. The relative values of the total energy of single S atom at the OIS and the TIS in nickel as a function of strain.

Fig. $5 \mathrm{~g}$-i presented the Bader charges of the $\mathrm{S}$ atom and neighboring $\mathrm{Ni}$ atoms for different strains. In all these cases, the Bader charge of $\mathrm{Ni}$ at the position of vertex was positive. The values are 0.26 e (a compressive strain of $-5 \%$ ), 0.26 e (without strain $0 \%$ ) and 0.25 e (a tensile strain of $+5 \%$ ), respectively. The interstitial $S$ atom obtains the electron from the host $\mathrm{Ni}$ atom at the position of vertex and becomes negatively charged, with a Bader charge of approximately $-0.05 \mathrm{e}$ (Fig. $5 \mathrm{~h}$ ). Such value increase to -0.01 e for a compressive strain of $-5 \%$ (Fig. $5 \mathrm{~g}$ ) but decreases to -0.07 e for a tensile strain of $+5 \%$ (Fig. $5 i$ ), consistent with the results that the tensile stress may enhance the bonding between $\mathrm{Ni}$ and $\mathrm{S}$ atoms from the valence charge density distribution analysis. In other words, the tensile stress promoted structure stability, whereas the compressive stress promoted structure instability. The Bader charges of $\mathrm{S}$ and $\mathrm{Ni}$ at the position of vortex were negative. The system is meeting the charge transfer conservation. This calculated Bader charge also revealed that $\mathrm{S}$ induced a slight reduction in the directionality of bonding between $\mathrm{Ni}$ atoms. Considering that the electron density everywhere in the $\mathrm{Ni}$, as well as in most metals, was much higher than this value, we should find lowerelectron density sites with lower binding energy. In $\mathrm{Ni}$, the present results showed that the tensile stress had an electron density of -0.07 e.

\subsection{Diffusion of single $S$ in Ni under stress}

We studied the diffusion energy barrier of $\mathrm{S}$ in $\mathrm{Ni}$ as a function of strain. Under applied stress, the most stable site in Ni was still OIS. Thus, we directly examined the diffusion barrier between two neighboring OIS (O-O path) as well as the diffusion barrier through the $\mathrm{O}-\mathrm{T}-\mathrm{O}$ path.

With the applied $z$-axis stress, the diffusion energy profile and the corresponding diffusion paths are shown in Fig. 6, where the energy is referenced to that for $\mathrm{S}$ at OIS. As shown in Fig. 6, the profiles have a maximum at site 3 via the $\mathrm{O}-\mathrm{O}$ path and have a maximum at site 8,12 via the $\mathrm{O}-\mathrm{T}-\mathrm{O}$ path; therefore, the migration barriers for a single $\mathrm{S}$ via the $\mathrm{O}-\mathrm{O}$ and $\mathrm{O}-\mathrm{T}-\mathrm{O}$ paths are equal to the maximum energy values minus the minimum energy values in each diffusion path. When without the applied stress on the system, the diffusion barrier was calculated to be 28.5284 and $14.7380 \mathrm{eV}$ via the $\mathrm{O}-\mathrm{O}$ and $\mathrm{O}-\mathrm{T}-\mathrm{O}$ paths, respectively. This finding proves that S would still go back to the center of OIS and would choose the TIS for the middle path via the $\mathrm{O}-\mathrm{T}-\mathrm{O}$ path if the $\mathrm{S}$ atom was slightly off the center of TIS or OIS. Thus, TIS was the local energy minimum site for $\mathrm{S}$. The diffusion energy barrier as a function of strain for both $\mathrm{O}-\mathrm{O}$ 

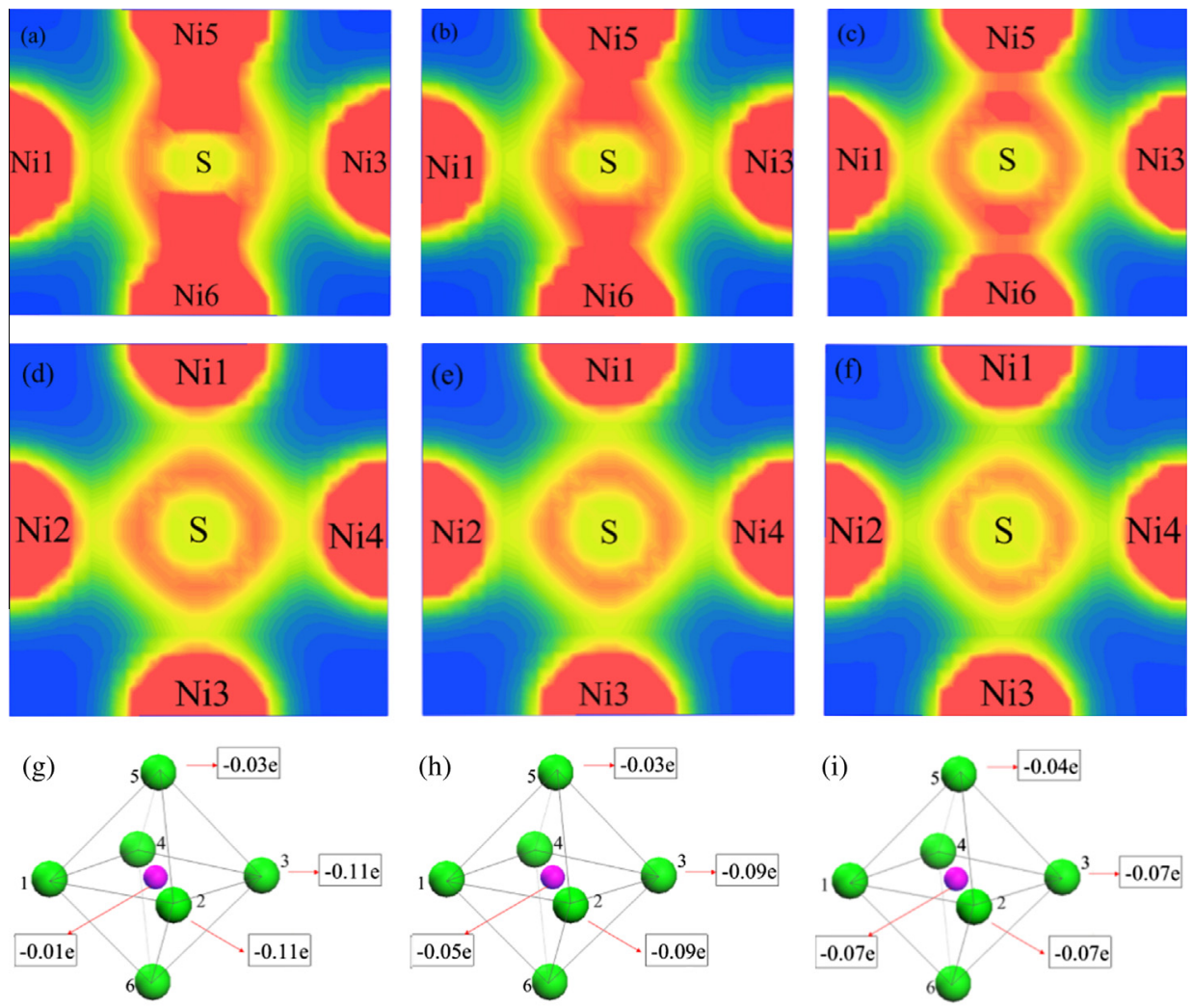

(j)
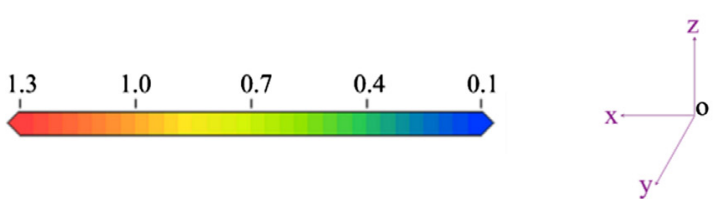

Fig. 5. The valence charge density distribution of the (020) plane (a-c), the (002) plane (d-f) and the Bader charges ( $\mathrm{g}-\mathrm{i})$ of $\mathrm{S}$ at OIS in nickel for different strains ( $-5 \%, 0$ and $+5 \%)$, Isosurface values: from 0.1 to 1.3 .

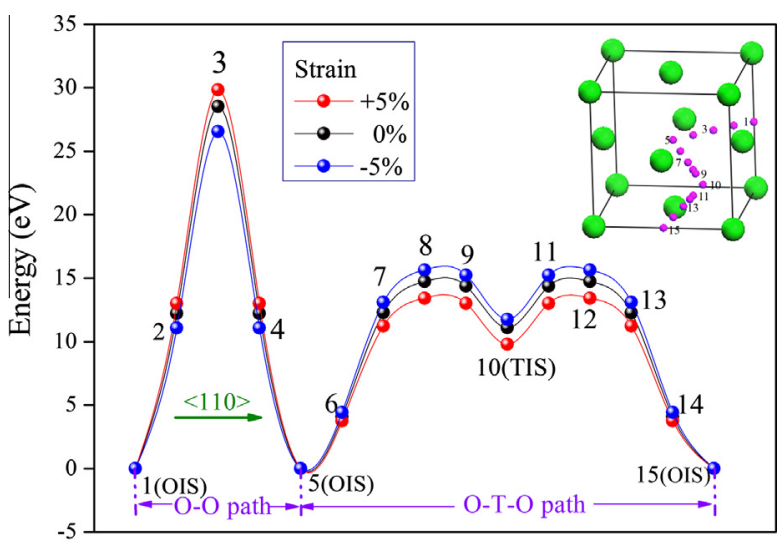

Diffusion path

Fig. 6. Diffusion energy profile of $\mathrm{S}$ atom via $\mathrm{O}-\mathrm{O}$ path and $\mathrm{O}-\mathrm{T}-\mathrm{O}$ path in nickel under the $z$-axis strain $(-5 \%, 0$, and $+5 \%$.

and $\mathrm{O}-\mathrm{T}-\mathrm{O}$ paths are shown in Fig. 7. With increasing tensile stress, the diffusion barrier of single $\mathrm{S}$ for the $\mathrm{O}-\mathrm{O}$ path became higher and for the $\mathrm{O}-\mathrm{T}-\mathrm{O}$ path became lower. Within the strain range of $-5 \%$ to $+5 \%$, the diffusion barrier via the $\mathrm{O}-\mathrm{O}$ path increased from $26.5540 \mathrm{eV}$ to $29.8300 \mathrm{eV}$ compared with $28.5285 \mathrm{eV}$ for the zero strain. Meanwhile, the barrier via the O-T-O path decreased from $15.6410 \mathrm{eV}$ to $13.4250 \mathrm{eV}$ compared with $14.7380 \mathrm{eV}$ for the zero strain. The diffusion energy barrier via the $\mathrm{O}-\mathrm{O}$ path increased because the path was parallel to the $\langle 110\rangle$ direction when the $z$-axis

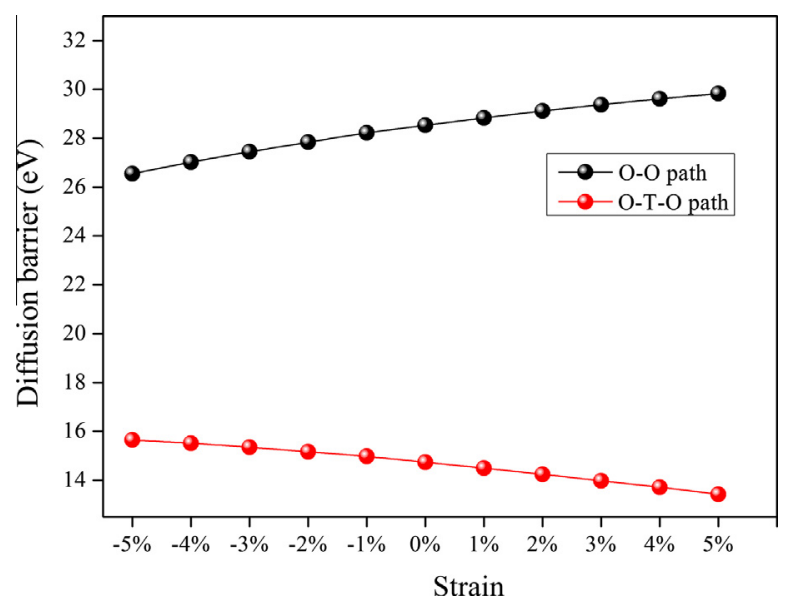

Fig. 7. Diffusion energy barriers of $\mathrm{S}$ via the $\mathrm{O}-\mathrm{O}$ path and $\mathrm{O}-\mathrm{T}-\mathrm{O}$ path as a function of applied strain.

tensile stress was applied. By contrast, it was compressed in the (002) plane, leading to less vacancy. The diffusion energy barrier via the $\mathrm{O}-\mathrm{T}-\mathrm{O}$ path decreased because the increasing tensile strain provided more vacancy. The vacant space for $\mathrm{S}$ extended the $\mathrm{Ni}-\mathrm{S}$ bonds. The Ni-S bonding thus became weaker, giving rise to the lower $\mathrm{S}$ diffusion barrier. Furthermore, the diffusion barrier via the $\mathrm{O}-\mathrm{T}-\mathrm{O}$ path was lower than that via the $\mathrm{O}-\mathrm{O}$ path. In conclusion, the $\mathrm{S}$ atom would still prefer to jump via the $\mathrm{O}-\mathrm{T}-\mathrm{O}$ path. 


\section{Conclusions}

To understand the stability and diffusion behavior of $\mathrm{S}$ in $\mathrm{Ni}$ under stress, we investigated the structure of S in Ni using firstprinciples methods. According to the calculated formation energy and binding energy, sulfur atoms easily formed as impurity atoms in the Ni matrix, causing greater thermodynamic instability compared with pure Ni. The diffusion barrier of single $\mathrm{S}$ decreased with increasing tensile stress. The compressive stress caused structure instability. S diffusion was accelerated under the applied stress, which is disadvantageous in suppressing $\mathrm{S}$ retention in Ni. These results indicate that dissolved $\mathrm{S}$ still has a tendency to precipitate from the Ni matrix even at a low concentration to increase the stability of the system further. This result may explain the experimental observation that sulfur segregation to grain boundary.

\section{Acknowledgments}

This research was supported by the National Natural Science Foundation of China (Grant No. 51371123), the National Natural Science Foundation of Shanxi province (Grant No. 2014011045), the Research Fund for the Doctoral Program of Higher Education of China (20131402110003), Graduate Science and Technology Innovation Fund of Shanxi province (Grant No. 20133043) and Graduate Science and Technology Innovation Fund of Taiyuan University of Technology.

\section{References}

[1] R. Gilles, D. Mukherji, P. Strunz, Physica B 234 (1997) 1008-1010.

[2] G.J.S. Higginbotham, Mater. Sci. Technol. 2 (1986) 442-460.

[3] H. Esaka, M. Tamura, K. Shinozuka, Mater. Trans. 44 (2003) 829-835.
[4] R.C. Reed, The superalloys fundamentals and applications, Cambridge University, 2006.

[5] D.E. Kim, S.L. Shang, Z.K. Liu, Comput. Mater. Sci. 47 (2009) 254-260.

[6] M.J.V. Setten, G.A.D. Wijs, Phys. Rev. B 76 (2007) 075125.

[7] A.R. Akbarzadeh, C. Wolverton, V. Ozolins, Phys. Rev. B 79 (2009) 184102.

[8] F.D. Torre, H.V. Swygenhoven, M. Victoria, Acta Mater. 50 (2002) 3957-3970.

[9] S. Kobayashi, S. Tsurekawa, T. Watanabe, G. Palumbo, Scripta Mater. 62 (2010) 294-297.

[10] J.K. Heuer, P.R. Okamoto, N.Q. Lam, J.F. Stubbins, J. Nucl. Mater. 301 (2002) 129-141.

[11] H.P. Chen, R.K. Kalia, E. Kaxiras, G. Lu, A. Nakano, K.I. Nomura, A.C.T.V. Duin, P. Vashishta, Z. Yuan, Phys. Rev. Lett. 104 (2010) 155502.

[12] M. Yamaguchi, M. Shiga, H. Kaburaki, Mater. Trans. 47 (2006) 2682-2689.

[13] W.T. Geng, J.S. Wang, G.B. Olson, Science 309 (2005) 1677c.

[14] L.G. Wang, C.Y. Wang, Comput. Mater. Sci. 11 (1998) 261-269.

[15] M.J.N.V. Prasad, A.H. Chokshi, Acta Mater. 58 (2010) 5724-5736.

[16] I. Matsui, T. Uesugi, Y. Takigawa, K. Higashi, Acta Mater. 61 (2013) 3360-3369.

[17] M.G. Lozinskiy, G.M. Volkogen, N.Z. Pertsovskiy, Russ. Metall. 5 (1967) 65.

[18] D. Boutassouna, R.L. Gall, I.K. Lefkaier, Metal 5 (2012) 23-25.

[19] H.H. Kart, M. Uludogan, T. Cagin, Comput. Mater. Sci. 44 (2009) 1236-1242.

[20] Y. Jiang, R. Liu, Scripta Mater. 62 (2010) 782-785.

21] H. Sawada, Comput. Mater. Sci. 55 (2012) 17-22.

[22] M. Allart, F. Christien, R.L. Gall, P. Nowakowski, C.R.M. Grovenor, Scripta Mater 68 (2013) 793-796.

[23] H. Li, S. Xia, W.Q. Liu, T.G. Liu, B.X. Zhou, J. Nucl. Mater. 439 (2013) 57-64.

[24] B. Beeler, B. Good, S. Rashkeev, C. Deo, M. Baskes, M. Okuniewski, J. Nucl. Mater. 425 (2012) 2-7.

[25] M.D. Segall, P.J.D. Lindan, M.J. Probert, C.J. Pickard, P.J. Hasnip, S.J. Clark, J Phys.: Condens. Matter 14 (2002) 2717.

[26] C.C. Fu, F. Willaime, P. Ordejon, Phys. Rev. Lett. 92 (2004) 175503.

[27] N. Chetty, M. Weinert, T.S. Rahman, J.W. Davenport, Phys. Rev. B 52 (1995) 6313-6326.

[28] J. Jorritsma, J. Mydosh, J. Appl. Phys. 84 (1998) 901-906.

[29] M. Hajjaji, J. Alloys Compd. 274 (1998) 185-188.

[30] G.H. Lu, S.H. Deng, T.M. Wang, Phys. Rev. B 69 (2004) 134106.

[31] G. Henkelman, A. Arnaldsson, H. Jonsson, Comput. Mater. Sci. 36 (2006) 354360.

[32] E. Sanville, S.D. Kenny, R. Smith, J. Comput. Chem. 28 (2007) 899-908.

[33] Y.L. Liu, Y. Zhang, H.B. Zhou, G.H. Lu, F. Liu, G.N. Luo, Phys. Rev. B 79 (2009) 172103. 